\title{
Critical success factors in planning for Web services in the financial services industry
}

\author{
H. Howell-Barber ${ }^{1} \&$ J. Lawler ${ }^{2}$ \\ ${ }^{1}$ Executive Advisory Board of Ivan G. Seidenberg School of Computers, \\ Science and Information Systems, Pace University, USA \\ ${ }^{2}$ Information Systems Department, Pace University, USA
}

\begin{abstract}
As increasingly more firms in the financial services industry expand their use of Web services, and as others begin to adopt services, understanding the planning requirements for this technology becomes increasingly critical for business managers and technologists. This study explores a generic methodology of a Web services plan that can be used to accelerate accurate project planning, helping to avoid project planning becoming a major project in itself. This study identifies critical success factors that contribute effectively to the planning success of Web services projects in the financial services industry. The study furnishes a methodology model for the features of such a plan, by identifying components that can be reused and refined safely for a small inter-departmental project, a medium cross-departmental project, and a large inter-firm project. Business and methodological factors are indicated to be more important than technological factors in the success of the projects, though technology is reviewed in the study, and implications include planning recommendations, as they relate to Web services. This study will benefit management practitioners, researchers and technologists in the successful planning for Web services in the financial services industry.

Keywords: BPEL4WS, business process, project plan, service description, service-oriented architecture, SOA, UDDL, Web services, XML and WSDL.
\end{abstract}

\section{Background}

A Web service communicates using Simple Object Access Protocol (SOAP) messages over HyperText Transfer Protocol (HTTP). Services are published and 
described in a Universal Description, Discovery and Integration (UDDI) registry, using Web Services Descriptor Language (WSDL). Clients search the registry for services using SOAP messages. Messages that cross firewalls may be secured with Web Services Security (WS-S). Web services implementations parallel the client/server paradigm (Ma [1]), except that components use text-based Extensible Markup Language (XML) and share open standards and cross-vendor support. SOAP is from the World Wide Web Consortium (W3C), and WSDL, UDDI, and WS-S are from the Organization for the Advancement of Structured Information Standards (OASIS), whose members include all the major software vendors. HTTP, the de facto standard for connecting servers on the Web, is defined by RFC 1945 of the Internet Engineering Task Force (IETF).

A Service Oriented Architecture (SOA) provides loosely coupled services that expose business functions with published discoverable interfaces (Adams et al. [2]). An enterprise SOA leverages business logic and data from existing systems to support flexibility and adaptability of changing business environments of systems. SOA services map to business entities, allowing enterprise integration on the business level (Krafzig et al. [3]). Web services may be implemented as the first step to SOA; however, it is possible to have an SOA without Web services. The additional layer of abstraction in Web services allows authorized users access to information on heterogeneous native platforms. Services, discovered in legacy applications or created anew, may be combined into new services, using Business Process Execution Language for Web services (BPEL4WS), also from OASIS.

Businesses are being pushed to explore SOA architectures to avoid missing competitive advantages, while vendors race to produce or upgrade products that support these specifications. The additional layer of abstraction, new technology, and limited timeframes make planning for Web Services and SOA simultaneously more critical and more complicated. This study explores techniques for handling the added complexity, by highlighting tasks specific to Web services projects, and providing recommendations for using prior project experience to facilitate scheduling activities. The suggestions are vendor-neutral.

\section{Introduction}

The manager responsible for Web services project initiation must ensure that the business leads the project. Business participation is the most important factor in the success of an SOA (Lawler et al. [4]). Sponsors (business, customer, and technology) will be identified. Advisory groups with representatives from business, customer and technology areas will be established for the project. Stakeholders (individuals not directly involved in the project, but who can affect the outcome of the project) will also be identified by the groups. Regular advisory group and stakeholder meetings will be scheduled by the groups. An exercise to assess the organization's tolerance for change must be completed with the assistance of the advisory group. Remedial actions may be taken if the organization is change-averse. 


\subsection{SOA governance}

A governance board (with business, customer and technology participants) will guide the SOA implementation across a range of Web services projects. It will (a) maintain communication among SOA participants and stakeholders, (b) establish service access rules, (c) define business goals and performance indicators, (d) define an approach for modeling business processes, (e) establish service quality reviews, (f) document assumptions included in service requirements, (g) promote the cultural changes required for SOA success, (j) establish a process for business component identification, (k) establish a process for service prioritization, and (l) establish processes for lifecycle management and versioning (Bieberstein et al. [5]).

\section{$3 \quad$ Methodology model}

It is important to think big, but start small. It is useful to identify an entire business segment that can benefit from SOA, but the pilot project should deliver a few Web services in six months or less (Knorr and Rist [6]). Each pilot activity will lay the foundation for advancement toward the implementation of a true SOA. When the first deliverables successfully address an obvious business problem, they help to ensure approval and funding of larger projects.

\subsection{Small (pilot) project (intra-departmental)}

The pilot project (3-6 months) will address a critical business requirement, while ascertaining a technology and planning approach. For example, it could combine digital images of signed trust documents with customer data in a banking operations area. The seemingly disproportionate number of management tasks in the project plan furnishes a framework for future projects.

\subsection{Medium project (inter-departmental)}

A medium-sized project (6 months to a year) could involve rolling out a set of processes across operations areas in the same firm. An example is if the same trust documents were made available to the compliance monitoring area along with additional services that provide historical transaction activity. The SOA governance team will begin to exercise its mandate. The plan for the medium project will include additional coordination, requirements gathering (including the creation of a UDDI registry), and technology tasks. A carefully maintained project history will assist future (larger) projects.

\subsection{Large project (parent firm and select subsidiaries)}

A large project (a year or longer) will lead to the creation of a full SOA. This project could provide an expanded set of processes to selected subsidiaries of the financial services firm. The SOA governance role must be fully activated. While considering all tasks in the extensive list of activity details at 
www.hbink.com/webservicesplan, project communications, service security and cross-platform compatibility will be critical. A Web services glossary is furnished for further study. Prior project history, lessons learned, and best practices from earlier projects will be critical.

\section{$4 \quad$ Analysis of model}

Table 1 furnishes a high level outline of Web services plan activities. The Group Sequence column suggests an order for the initiation of planning activities. Activities with the same Group Sequence may begin in parallel. Groups 2-9 occur throughout the project lifecycle. Activities ramp up at the beginning of requirements, analysis and design, and development/implementation. Testing requirements are refined during analysis and design and performed during implementation.

Table 1: Web services planning activity groups.

\begin{tabular}{|l|c|l|c|}
\hline \multicolumn{1}{|c|}{ Activity Group } & $\begin{array}{c}\text { Group } \\
\text { Sequence }\end{array}$ & \multicolumn{1}{c|}{ Activity Group } & $\begin{array}{c}\text { Group } \\
\text { Sequence }\end{array}$ \\
\hline Methodology & 0 & Requirements & $10 . \mathrm{a}$ \\
\hline Project Initiation & 1 & Security & $10 . \mathrm{b}$ \\
\hline Project Process & 2 & Testing $^{* *}$ & $10 . \mathrm{c}$ \\
\hline Project Communications & $3 . \mathrm{a}$ & Project Change Management & 11 \\
\hline Project Planning & $3 . \mathrm{b}$ & Analysis and Design & $12 . \mathrm{b}$ \\
\hline $\begin{array}{l}\text { Role Assignment and } \\
\text { Confirmation }\end{array}$ & $3 . c^{*}$ & Architecture & $12 . \mathrm{a}$ \\
\hline Risk Management & 4 & Development/Implementation & 13 \\
\hline Best Practices & 5 & Deployment & $14 . \mathrm{a}$ \\
\hline Problem Management & 6 & Management and Monitoring & $14 . \mathrm{b}$ \\
\hline Procurement Management & $7^{*}$ & Project History & 15 \\
\hline Human Resources & $8^{*}$ & Sunset & 16 \\
\hline Training & $9^{*}$ & & \\
\hline
\end{tabular}

Activity ramps up at the beginning of requirements, analysis and design, and development / implementation.

${ }^{* *}$ Refined during design; performed during implementation.

\subsection{Methodology}

Methodology factors are important to the success of an SOA project (Lawler et al. [4]). Including service orientation into project management assumes selection of an established methodology that will be enhanced to include service-related tasks. Complexity and changing business requirements will require iterative development. For the strategically important SOA, the methodology will tend toward the side of the heavyweight processes (Krafzig et al. [3]).

\subsubsection{Project initiation}

Project initiation must include creation and syndication of a strong business case. Business opportunities and potential benefits will be analyzed, prioritized and used as input to statements of scope, objectives, and goals. The initial 
cost/benefits analysis will be performed (with clearly documented assumptions). Sign-off and funding authority for the overall project and major project checkpoints (initiation, requirements, analysis and design, testing, and deployment) will be identified by the project director. Sign-off at each major checkpoint will be required and will include funding for the subsequent activities.

After sign-off of initiation, an experienced manager for the remainder of the project will be assigned by senior managers. Her first activity will be to create a vision statement from the scope, objectives, and goals documents. A kick-off presentation will include the vision statement and, optionally, a prototype to help demonstrate proposed project deliverables.

\subsubsection{Project process}

The project process must include requirements management, project planning, tracking, oversight, quality assurance, and configuration management, in order to produce repeatable results (Level 2 in the software Capability Maturity Model (CMM)) (Leffingwell and Widrig [7]). Quality is important as mistakes will require changes to a larger number of project artifacts. Therefore, the requirements and analysis and design activities should take at least $60 \%$ of the project effort. As projects move toward true SOA, plans will include defined process features (CMM Level 3), such as cross-organization process, a formal training program, integrated software management, product engineering, intergroup coordination, and peer reviews. Measurement and monitoring of the process itself (CMM Level 4) will support inclusion of successful process features into future projects. Establishing a naming standard for project artifacts helps organize the main sections of the project and enables easy referencing by team members and clients throughout the project. An adaptable process with appropriate enforcement mechanisms will help to ensure that the project processes themselves are as non-intrusive as possible (Goto and Colter [8]).

\subsubsection{Project communications and project information center}

If all software builds took no time, and development was perfect, the limiting factor in project success would probably be communications (Doar [9]). Communication is facilitated by building a standard terminology used across the business and technical communities (Bieberstein et al. [5]). Thus, a communications plan and processes will be established early in the activity sequence. A Project Information Center will furnish a foundation for the communications plan and processes. This single (virtual) source will have secured, role-based access, with an index and pointers to (a) all project communications, (b) project process, (c) project plans and planning archives, (d) startup, requirements, analysis, design and architecture documents, (e) a project glossary with industry specific XML schema (e.g., FinXML, FIXML), and a taxonomy of business terminology specific to the firm and technology terms specific to the project, (f) contact information, (g) development artifacts and version information (checked in daily), (h) project samples, (i) status reports, (j) issues tracking and problem management files, (k) risk management 
documents, and (1) change control procedures. Maintenance procedures will include daily backups and the ability to achieve full recovery in half a day.

\subsubsection{Project planning}

The project manager will create a plan to track a greater number of tasks and corresponding roles (Table 2), even on small projects. The work breakdown structure (WBS) will include a larger number of artifacts to be developed and tracked by her. Greater involvement of the customer community requires coordination of individual activities outside the manager's official organization. Task scheduling will challenge both the manager and activity owners to furnish estimations for tasks they have never performed or observed, using new software, and in new environments. Complex or unfamiliar activities will need higher priority and may be scheduled ahead of their usual sequence to give team participants more time to resolve unexpected problems. Smaller deliverables will furnish beneficial results in a shorter timeframe. For example, deliverables may be scheduled weekly for small projects, every two weeks for medium projects, and monthly for larger projects. The project manager will help himself and future project managers by maintaining a detailed planning archive. Each plan change and reasons for that change will be recorded for future reference and problem avoidance. In successive projects, the project manager may find estimation assistance in his own plans or in the plans of his predecessors. The plan will be well syndicated, with multiple copies in a highly visible location to promote awareness and compliance.

\subsubsection{Role assignment and confirmation}

Table 2 lists the roles associated with a Web services project [10]. Most traditional roles will be expanded, several roles will be added, and user roles modified to take advantage of new services. Responsibilities of each role will be defined, assigned, and confirmed by the manager. Training requirements will be identified by the manager.

Table 2: $\quad$ Web services / SOA project roles.

\begin{tabular}{|c|c|}
\hline $\operatorname{Architect~}^{(*)}$ & Project administrator ${ }^{(*)}$ \\
\hline Business analyst ${ }^{(*)}$ & Project manager $^{(*)}$ \\
\hline Business testers $^{(*)}$ & Security specialist ${ }^{(*)}$ \\
\hline Change process manager ${ }^{(*)}$ & Service developer ${ }^{(+)}$ \\
\hline Configuration manager $^{(*)}$ & Service modeler $^{(+)}$ \\
\hline Database administrator $^{\left({ }^{*}\right)}$ & Services librarian/governor ${ }^{(+)}$ \\
\hline Deployment team $^{(*)}$ & SOA architect ${ }^{(+)}$ \\
\hline Developers ${ }^{(*)}$ & Systems administrator ${ }^{(*)}$ \\
\hline Facilitators ${ }^{(*)}$ & Technical writer \\
\hline Governance team $^{(+)}$ & Test manager ${ }^{\left({ }^{*}\right)}$ \\
\hline Interoperability tester ${ }^{(+)}$ & Tool administration ${ }^{(*)}$ \\
\hline Knowledge transfer facilitator ${ }^{(*)}$ & Toolsmith $^{(*)}$ \\
\hline Legacy adaptation specialist ${ }^{(+)}$ & UDDI administrator $^{(+)}$ \\
\hline Network administrator ${ }^{(*)}$ & User Roles ${ }^{(\nexists)}$ \\
\hline Process flow designer (Optional) ${ }^{(+)}$ & Vendor interface ${ }^{(*)}$ \\
\hline
\end{tabular}

* Expanded Roles

+ New Roles

$\neq$ Modified Roles 


\subsubsection{Risk management}

Risk management receives greater emphasis because of exceptional challenges associated with objective business process extraction, resistance to change, information gaps within and among business and technology communities, implementation of new vendor products, new and updated industry standards, staffing requirements, environmental complexity, and absence of cooperation across business silos. Risks and possible countermeasures will be listed, evaluated, prioritized, and tracked by management.

\subsubsection{Best (and worst) practices - patterns and antipatterns}

Patterns are collections of best practices from the combined experiences of industry specialists. Patterns for e-Business are a group of reusable assets that can help speed the process of developing Web-based applications. They include business, integration, application, runtime, and combination patterns [11]. Since more than $80 \%$ of projects fail or run over budget, antipatterns are an even greater area to be mined for problem avoidance techniques (Ang et al. [12]). They prevent problems by identifying frequently recurring errors.

\subsubsection{Problem management}

Though problem management is normally associated with testing, it receives its own heading because problems that occur outside the testing activity must also be managed by management. A process including problem capture, evaluation, categorization, prioritization, resolution, and reporting will be implemented and enforced by management. A history of problems and their resolutions will be maintained to help future teams avoid similar problems.

\subsubsection{Procurement management}

A structured procurement process will ameliorate risks by helping ensure that vendors and products adequately support project objectives. Vendor selection criteria will include staff quality, responsiveness, short-term support capabilities, and the probability that they will be able to support future requirements. Product selection will include processes for installation and rigorous in-house testing before purchase agreements are signed by management. If specialist consultants are required on the project, there must be clearly stated performance objectives and willingness to make adjustments if the objectives are not met by the consultants.

\subsubsection{Human resources}

These activities include identification of skills requirements, skills assessments, and identification of training needs. If there is no time to train existing staff, it may be necessary to hire additional staff (from approved vendors), including contract developers or specialist consultants. If this is the case, new staff orientation procedures must be in place (to help them "hit the ground running"). Orientation will include physical access rules, development environment access, equipment, telephone (if functioning on premises), connection capabilities (if not functioning on premises), personnel introductions, business introductions, firm 
orientation, and a technology overview. Support and counseling will help ensure optimal performance.

\subsubsection{Training}

The leaders of the SOA revolution will be business personnel who understand technology and technology personnel who understand the business (McKean [13]). Therefore, appropriate training and cross-training are critical in both business and technology spheres. Training of business analysts to identify and model necessary business services is the next item of importance in the success to an SOA, followed by appropriate technology training.

\subsubsection{Requirements}

Requirements for a Web services or SOA project must be more clearly defined than for prior implementations. The business and customers should drive the activity. Business analysts must help to determine which services (spanning organizational boundaries) need priority and which processes will be included in the services (including the possibility of combining processes from multiple applications within the same service), determine what data will be included in the services, and (most critically) how the data will be named in the system. Defining the business meaning of transactions and data is the most intractable issue that systems managers face in the system (Sleeper [14]). Proportionately more time will be spent in requirements gathering and specification of services, in order to ensure that business participants agree among themselves on terminology, scope, goals, and priorities. Service Level Agreements (SLA) will be included in the requirements. Acceptance criteria will be clearly defined by management.

\subsubsection{Security}

Though important in financial services, security will have an extra layer of complication when users from different business groups begin to access the same services. Though identification, authentication, authorization, integrity, confidentiality, auditability, and non-repudiation should be considered as part of requirements, Web service tools may fail to support all requirements (Van de Putte et al. [15]). Because Web Services Security (WS-S-April, 2004) and Security Assertions Markup Language (SAML-March, 2005) are new, team members will start early evaluations to ensure that security products provide the appropriate level of security, while conforming to industry standards. Though WS-S indicates how to use previously existing security technologies within the Web services environment, achieving the right mixture of features takes significant time and effort (Newcomer and Lomow [16]). Finally, to avoid delays in development, testing and deployment, role-based access to project artifacts (old and new), services and services components will be defined by management.

\subsubsection{Testing}

Testing requirements and test cases should be identified along with requirements for processes and services. The test plan will be finalized during analysis and 
design, and will start to be implemented during development and implementation. Though this sequence for testing requirements is not new for Web services, it is important that testing activities occur early and often. Business functionality should be as clear as possible before testing of Web services begins for the system.

\subsubsection{Project change management}

Appropriate attention to requirements, analysis and design will help decrease the need for changes. However, a major justification for SOA implementation is rapid support for inevitable changes in the business environment. Therefore, there must be a process for gathering, reviewing, prioritizing, and signing-off to project changes that is as rigorous as the requirements and design efforts. A clearly defined process for version control will be followed by management. Change management for underlying legacy components will be included in the process. Changes at the business and process level will be controlled by the governance team. Business participants will be aware of the effect changes will have on project timetables and budget before they sign off on changes.

\subsubsection{Analysis and design}

As with the requirements, business participation will be more critical than before in non-SOA projects. Analysis of existing applications will identify candidate functions and data. Redundant functions and data will be flagged as candidates for sunset activities after the successful project completion. Where possible, industry schemas will be used by management. Because the WSDL is the contract between the developer of the service and the user of the service, it is important to design the WSDL first before developing the service. The framework for Web services management should also be included in the design.

\subsubsection{Architecture}

The technology facing members of the team will begin evaluating existing environments against requirements as soon as a first draft of the requirements is available. The architect will furnish feedback to the requirements team regarding what is feasible, given the state of the technology, and will ultimately recommend an environment that will address the finalized requirements. After approvals and corresponding funding, she will partner with the procurement team to acquire, install, and test product upgrades or new products. To prepare for success as the organization moves toward a complete SOA, scalability will be included in the recommendation.

\subsubsection{Development/implementation}

Development will take a smaller proportion of project effort than with normal projects because of the increased efforts in requirements, analysis, and design. Coding standards are a good idea. At a minimum, team members should agree to code formatting guidelines and artifact naming standards. Development of project artifacts will occur within the framework of an agreed upon methodology that will include continuous integration (regular builds of the software, along with build tests). Strict version control of all artifacts within and across 
environments will begin with the first build. SOA-specific activities, such as legacy adaptations, as in service description and registry, should occur in parallel with the usual programming efforts to allow time for discovering and solving problems with the new technology. Error handling will be implemented and a list of error messages compiled by management.

\subsubsection{Deployment}

While final testing is being conducted, a rollout schedule addressing client orientation and role upgrades will be created, reviewed, and signed-off by management. A roll-out plan with necessary fallback steps will be created by management. Documentation will include a deployment diagram, deployment checklists, release documentation, system administration and general operations requirements (including recovery and failover plans).

\subsubsection{Management and monitoring}

Monitoring includes logging, tracing messages, security enforcement, and quality of service tracking (as specified in the requirements). Monitoring software will be evaluated and implemented during the testing cycles. Production monitoring will begin with the first deployment, using metrics and report layouts created in parallel with service design. Service level agreements specified in the requirements will be monitored by management. Potential effects on legacy systems will be reviewed by management.

\subsubsection{Project history as a reusable asset}

The plan will include project evaluation, project turnover, and process improvement (with critical input from the post implementation report). Documenting project history can help to develop better estimates and save planning time by leveraging templates from past projects [17]. This requires a strategy for recording project information across the team.

\subsubsection{Sunset}

A plan for eliminating duplicated systems, functions, data and overlapping projects, as discovered during analysis and design, will be created and reviewed by management. Redundancies will be eliminated in the process, as successively more services are implemented by management.

\section{Implications}

Immediate implications of this study include business benefits. Successful Service Oriented Architecture (SOA) will benefit from planned communications between business and technology departments that cooperate as partners. Business personnel will develop adequate knowledge of technology themes that will help in the implementation of intelligent changes. Technology personnel will have adequate knowledge of business topics that will help in improvements that are both technically and financially feasible. Training will be included to maintain the necessary knowledge. As a result, change-averse firms will become 
capable of further flexibility, as they discern benefits from changes of a Service Oriented Architecture.

Implications include increased capability and maturity of the information technology department. Departments that already have a bona fide methodology will expand and improve techniques, in order to manage increased complexity of a Service Oriented Architecture. Departments that do not have a methodology will institute one, in order to manage the projects. Planning will be critical in a methodology. Departments that institute change management processes will have probable and resultant successful Service Oriented Architecture projects.

Final implication of the study includes the criticality of initiating pilot projects in Service Oriented Architecture. Departments in information technology of firms have been successful in basic Web services projects and, in the main, have been developing advanced Service Oriented Architecture projects. Such projects furnish a foundation for practitioner and scholarly studies of potential benefits to firms that have not introduced the latter projects. Standards may be learned from best of class practitioner consultants and vendors that have helped firms in Service Oriented Architecture development and implementation of systems. Study indicates competitive advantage for fast follower and first mover firms that invest in the Service Oriented Architecture soon.

\section{Conclusion}

Appropriate planning will emphasize leadership from the business community. A sequence of plans, with each plan furnishing input to subsequent plans, will facilitate the implementation of Web services and migration to a full Service Oriented Architecture. Plans for medium and large-size developments will inherit successful sections from previous plans, while avoiding the problems discovered in earlier planning. Emphasis on elimination of typical project failure points will allow time for careful investigation and implementation of new development paradigms.

\section{References}

[1] Ma, K. J., Web services: What's real and what's not. IT Pro, March / April, p. 15, 2005.

[2] Adams, H., Gisolfi, D., Snell, J., \& Varadan, R., Best practices for Web services. IBM developerWorks, 1 November, 2002.

[3] Krafzig, D., Banke, K. \& Slama, D., Enterprise SOA: Service-Oriented Architecture Best Practices, Pearson PTR: Upper Saddle River, New Jersey, Online, 2004.

[4] Lawler, J., Anderson, D., Howell-Barber, H., Hill, J., Javed, N., \& Li, Z., A study of Web services strategy in the financial services industry. Information Systems Education Journal, 3(3), pp. 1-25, 2005.

[5] Bieberstein, N., Bose, S., Fiammante, M., Jones, K. \& Shah, R., Service Oriented Architecture Compass: Business Value, Planning, and 
Enterprise Roadmap, IBM Press (Pearson plc): Upper Saddle River, New Jersey, Online, 2006.

[6] Knorr, E. \& Rist, O., 10 steps to SOA. Infoworld, 7 November, p. 24, 2005.

[7] Leffingwell, Dean \& Widrig, D., Managing Software Requirements: A Unified Approach, Addison Wesley: Boston, p. 475, 2003.

[8] Goto, K. \& Colter, E., Workflow that Works, Second Edition, New Rider's Press: Indianapolis, Indiana, Online, 2004.

[9] Doar, M. B., Practical Development Environments, O'Reilly Media, Inc.: Sebastopol, California, Online, 2005.

[10] Web Services Project Roles, IBM developerWorks, Online www 128.ibm.com/developerworks/webservices/library/ws - roles/, 2004.

[11] IBM Patterns for e-Business, IBM developerWorks, http://www128.ibm.com/developerworks/patterns/, 2004.

[12] Ang, J., Cherbakov, L., \& Ibrahim, M., SOA anti-patterns. IBM developerWorks, November, 2005.

[13] McKean, K., Business-ification of IT. Infoworld, 23 May p. 8, 2005.

[14] Sleeper, B., The SOA puzzle: five missing pieces. Infoworld, 13 September, p. 42-51, 2004.

[15] Van de Putte, G., Jana, J., Keen, M., Kondepudi, S., Mascarenhas, R., Satish, O., Rudrof, D., Sullivan, K., \& Withinbank, P., Using Web Services for Business Integration, IBM Redbooks: San Jose, California, p. 33, 2004.

[16] Newcomer, E. \& Lomow, G., Understanding SOA with Web Services, Addison Wesley Professional: Boston, Online, 2004.

[17] Work Essentials for Project Managers: Using Historical Data to Improve Future Projects, http://office.microsoft.com/en-us/FX012217241033.aspx, 2004. 